\title{
The relation between color spreading and illusory contours
}

\author{
TAKEO WATANABE \\ Harvard University, Cambridge, Massachusetts \\ and \\ HIROSHIGE TAKEICHI \\ University of Tokyo, Tokyo, Japan
}

\begin{abstract}
In the present study, we examine the relation between neon color spreading (Redies \& Spillmann, 1981) and illusory contours. In Experiment 1, the effects of misalignment between the line elements on the illusory contours in the Ehrenstein figure and in the Redies-Spillmann figure were examined. The remarkable overlap of the two curves for the likelihood of perceiving illusory contours in the Ehrenstein figure and in the Redies-Spillmann figure suggests that the illusory contours surrounding brightness enhancement (Ehrenstein, 1941) and those surrounding neon color spreading are caused by the same mechanism. We further examined both the effects of the interposed grids seen either in front of or behind the figures (Experiment 2) and the effects of misalignment (Experiment 3 ) on the illusory contours and range of color spreading, and found a high correlation between the appearance/disappearance of illusory contours and global/local color spreading. In Experiment 4, we added new lines to induce illusory contours to the line elements inducing local color spreading. We found that global color spreading was seen to cover the area surrounded by the illusory contours. On the basis of these findings, we suggest that there is an interaction between illusory contours and local color spreading.
\end{abstract}

In the Ehrenstein figure (Ehrenstein, 1941), shown in Figure 1a, brightness enhancement surrounded by a circular illusory contour is observed in the empty gap formed by the four radially arranged black arms on a white background.

By inserting a colored cross in the empty gap in the Ehrenstein figure, as is shown in Figure 1b, Redies and Spillmann (1981) showed that the color of the inserted cross spreads outside of its containing contours and forms a circular area. This is called neon color spreading; it was first described by van Tuijl (1975) with a matrix pattern.

Recently, Watanabe and Sato (1989) have found that when the inserted cross or background and the arms in the Redies-Spillmann figure are nearly equiluminous, color spreading itself persists, but no clear contour defining the spreading is perceived. They state that the color appeared "to diffuse from the center" of the inserted cross, but that it had "no clear boundary," and faded "into its surroundings. The size of the spreading [was] ... about half of what it should be with the illusory con-

We thank Kazuo Nakatani and Shinsuke Shimojo for their suggestions regarding our research and manuscript, George L. Zimmerman and Katherine Morris for grammatical corrections, and two anonymous reviewers and $C$. $W$. Tyler for comments on the submitted manuscript. Part of this study was conducted while the first author was at the University of Tokyo. Requests for reprints should be sent to Takeo Watanabe, Department of Psychology, Harvard University, 1484 William James Hall, 33 Kirkland Street, Cambridge, MA 02138. tour" (Watanabe \& Sato, 1989, p. 429). That is, color speading without illusory contours was observed. ${ }^{1}$

Watanabe and Sato (1989) suggest that the neon color spreading observed in the Redies-Spillmann figure is caused by two different mechanisms: a mechanism for illusory contours and a mechanism for color spreading. According to their hypothesis, the illusory contour seen around the neon color spreading disappears because there is no luminance difference between the arms of the inducing figure (see Figure 1b) and part of its surroundings (the inserted cross and/or the background), while color spreading itself persists with no clear illusory contour.

There are still two problems to be resolved. First, there is a tacit assumption in Watanabe and Sato's (1989) hypothesis that illusory contours around the brightness enhancement in the Ehrenstein figure and those around the color spreading in the Redies-Spillmann figure are caused by the same mechanism. To test this assumption, in Experiments 1 and 2 we therefore examined whether or not these contours are constrained in similar ways by the same stimulus parameters.

The second problem to be resolved is that the relation between the mechanism for color spreading and that for illusory contours is still unclear. Day (1983) demonstrated that the shape of the neon color spreading is determined by the locations of additional dots in the Koffka figure (Koffka, 1935), which suggests that the color spreading is limited by partially delineated borders along which il- 


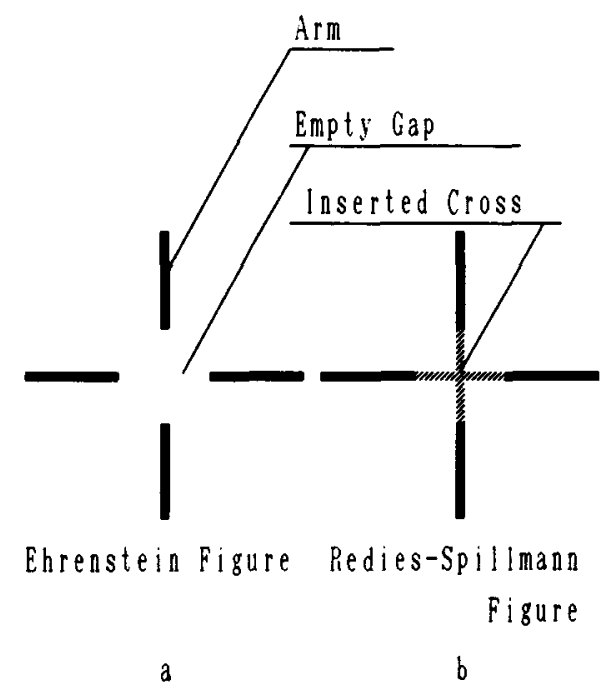

Figure 1. (a) Ehrenstein figure, in which a circular illusory contour is observed. (b) Redies-Spillmann figure, in which the color of the inserted cross is seen to spread out of its containing contour. The four lines constituting the Ehrenstein figure and the outer segments of the Redies-Spillmann figure may be called arms. We will call the space between the arms of the Ehrenstein figure the empty gap and the colored (represented as hatched) cross in the Redies-Spillmann figure the inserted cross.

lusory contours can form. Without such borders, the neon color is assumed to spread further.

According to Watanabe and Sato (1989), however, color spreading is locally confined to a small area when it is seen without any clear illusory contours under the equiluminous condition. If color spreading is more restricted with no clear illusory contours than it is with clear illusory contours, this finding cannot be completely accounted for by Day's (1983) hypothesis. Thus, in Experiments 2 and 3 we investigated whether or not there is any coincidence between a small size of color spreading and the disappearance of illusory contours. Since a correlation did occur, in Experiment 4 we further examined the possible interaction between the color spreading and illusory contours.

\section{EXPERIMENT 1}

\section{Method}

Subjects. Seven men and 1 woman, ranging in age between 23 and 31 years, participated as subjects. All had normal or correctedto-normal visual acuity. One of the authors served as a subject; the other subjects were not aware of the purpose of the experiment.

Materials. The stimuli were presented on a high-resolution CRT display (NEC PC-KD853, $640 \times 400$ pixels) of a microcomputer (NEC PC-9801 VX) at a distance of $57.3 \mathrm{~cm}$ from each subject's eyes. No fixation point was presented.

Examples of the stimuli are shown in Figures 2 and 3. Figure 2 contains modified Ehrenstein figures whose vertical and horizontal arms are misaligned horizontally ( $d x$ in length) and vertically ( $d y$ in length), respectively. When $d x=0$ and $d y=0$, the stimulus figure is an Ehrenstein figure.

Similarly, Figure 3 illustrates stimuli modified from the Redies-Spillmann figure. The vertical and horizontal lines of the crosses are misaligned horizontally ( $d x$ in length) and vertically ( $d y$ in length). When $d x=0$ and $d y=0$, the stimulus figure is a Redies-Spillmann figure that induces neon color spreading with illusory contours.

The size of the stimuli is shown in Figures 2 and 3. The color of the background was white. The luminance of the background

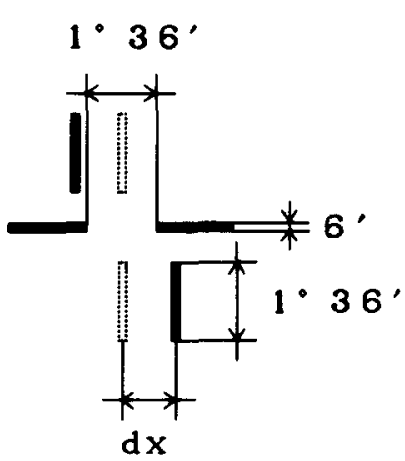

a

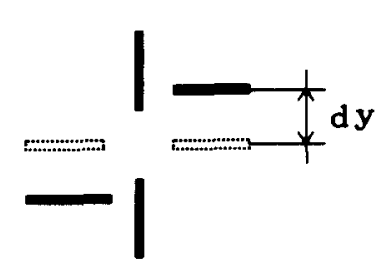

b

Figure 2. Examples of the stimuli used in Conditions 1 and 2 of Experiment 1. The vertical (a) and horizontal (b) arms of Ehrenstein figures are misaligned horizontally and vertically, respectively.

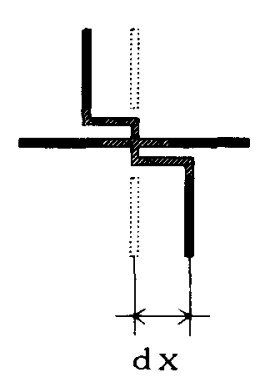

a

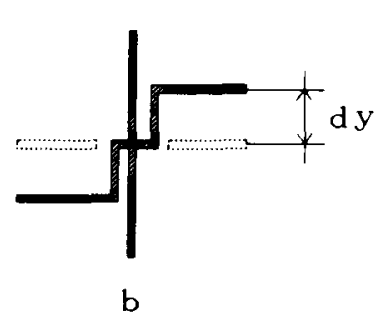

Figure 3. Examples of the stimuli used in Conditions 3 and 4 of Experiment 1. The vertical (a) and horizontal (b) arms of Ehrenstein figures are misaligned horizontally and vertically, respectively. Distorted colored crosses are inserted in the central gap, so as to connect both the misaligned and aligned arms of the Ehrenstein figures. The hatched lines represent the colored lines. 
was $32.5 \mathrm{~cd} / \mathrm{m}^{2}$. The misaligned arms of the figures were black. The inserted distorted crosses in the misaligned Redies-Spillmann figures in Figure 3 were blue ${ }^{2}(x=0.32, y=0.67$, luminance $=14.7 \mathrm{~cd} / \mathrm{m}^{2}$ ). When $d y=0, d x$ was changed from $0^{\circ}$ to $3^{\circ} 4^{\prime}$ in nine $23^{\prime}$ steps. When $d x=0, d y$ was changed similarly in nine steps. Thus, there were 17 stimulus patterns.

Procedure. The experimental session consisted of two conditions. In Condition 1, misaligned Ehrenstein figures with no crosses inserted into the empty gap, such as those shown in Figure 2a-b, were presented. On each trial, subjects had to report whether or not they saw an illusory contour connecting the end points of the four arms.

In Condition 2, misaligned Redies-Spillmann figures with colored crosses inserted, such as those shown in Figure 3a-b, were presented. On each trial, subjects had to report whether or not they saw the blue-green color spread within a clear illusory contour along the end points of the four arms.

On each trial, subjects had to respond "yes" or "no" by pushing the corresponding keys on the keyboard. There were no time limits. Each stimulus (each of the 17 combinations of $d x$ and $d y$ ) was presented six times. Thus, each condition consisted of $17 \times$ $6=102$ trials. Trials were presented in a random order for each subject.

The two conditions were presented to each subject in a random order. Experimental sessions were conducted in a dark room after dark adaptation for $10 \mathrm{~min}$.

\section{Results}

Mean positive response rates for each condition were plotted as a function of the amount of misalignment in Figure $4 .^{3}$ Subjects had to report whether or not they saw an illusory contour in the Ehrenstein figure (Condition 1: contour), and color spreading with illusory contours in the Redies-Spillmann figure (Condition 2: spreading). Both curves in Figure 4 indicate a clear tendency to decrease in response rates in each condition as the amount of misalignment increases. The response rates are nearly equal to each other for the whole range of misalignment.

The 50\% thresholds in each condition for each of two orientations (vertical and horizontal) of misalignment for

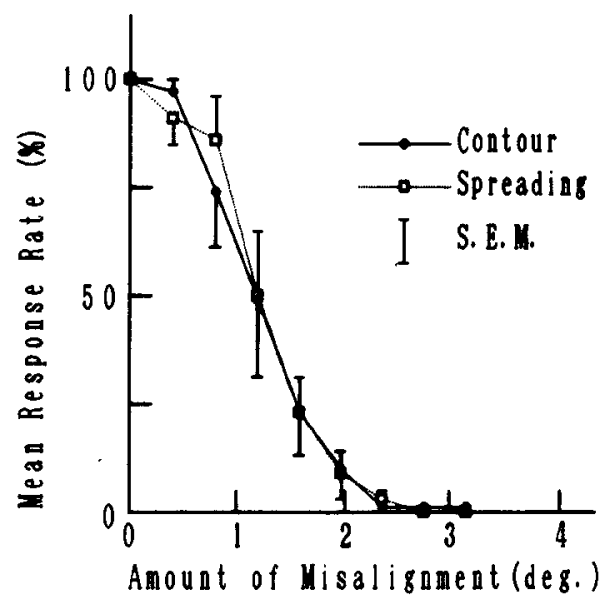

Figure 4. Mean positive response rates for the perception of (1) illusory contours in the Ehrenstein figures (Contour), and (2) color spreading with contours in Redies-Spillmann figures (Spreading), plotted as functions of the amount of misalignment. Length of each error bar, which is shown only on one side, represents one standard error of the mean (SEM). each subject were subjected to a two-way analysis of variance (ANOVA). The experimental conditions and the orientations of misalignment (vertical and horizontal) were the repeated factors. Neither effect was found to be significant.

\section{Discussion}

The remarkable overlap of the two curves representing the response rates and the nearly equal thresholds found in both conditions are compatible with the hypothesis that the illusory contours surrounding the brightness enhancement in the Ehrenstein figures and those seen surrounding color spreading in the Redies-Spillmann figures are caused by the same mechanism. However, one might still argue that the similar shape of the curves is not due to the same underlying mechanism for illusory contours, but to mere similarity between the figural structure of the misaligned Ehrenstein figures and that of the misaligned Redies-Spillmann figures.

One of the extraneous factors known to influence the appearance of illusory contours is the direction of apparent depth of the interposed grids composed of lines relative to the inducing figures. Rock and Anson (1979) observed that 20 out of 60 subjects perceived illusory contours when a modified Kanizsa figure (Kanizsa, 1955) was presented behind a grid of lines, whereas illusory contours were visible to none of 60 subjects when the Kanizsa figure was seen in front of the grid.

In Experiment 2, we interposed grids seen either in front of or behind the Ehrenstein figures and the RediesSpillmann figures, to examine whether or not the appearance of illusory contours seen in the Redies-Spillmann figures coincides with the appearance of illusory contours seen in the Ehrenstein figures when the apparent depth of these figures relative to the grids changes in direction. ${ }^{4}$

If an extraneous factor such as the apparent depth of the interposed grids, which is manipulated in Experiment 2, affects illusory contours in the Ehrenstein figures and those in the Redies-Spillmann figures in similar ways, the results cannot be attributed to the mere similarity of the figural structure. It would be more probable that both contours are caused by the same mechanism than that the independent variables manipulated in Experiments 1 and 2 , which are different in quality, similarly affect the two different mechanisms for the two different illusory contours.

In addition, it is worth mentioning that in Experiment 1, the subjects reported that color spreading was more restricted without illusory contours than it was with illusory contours. Thus, in Experiment 2, the relation between the appearance of global color spreading over large areas and the appearance of illusory contours was also examined.

\section{EXPERIMENT 2}

\section{Method}

Subjects. Twelve men, ranging in age between 21 and 34 years, participated as subjects. All had normal or corrected-to-normal visual 

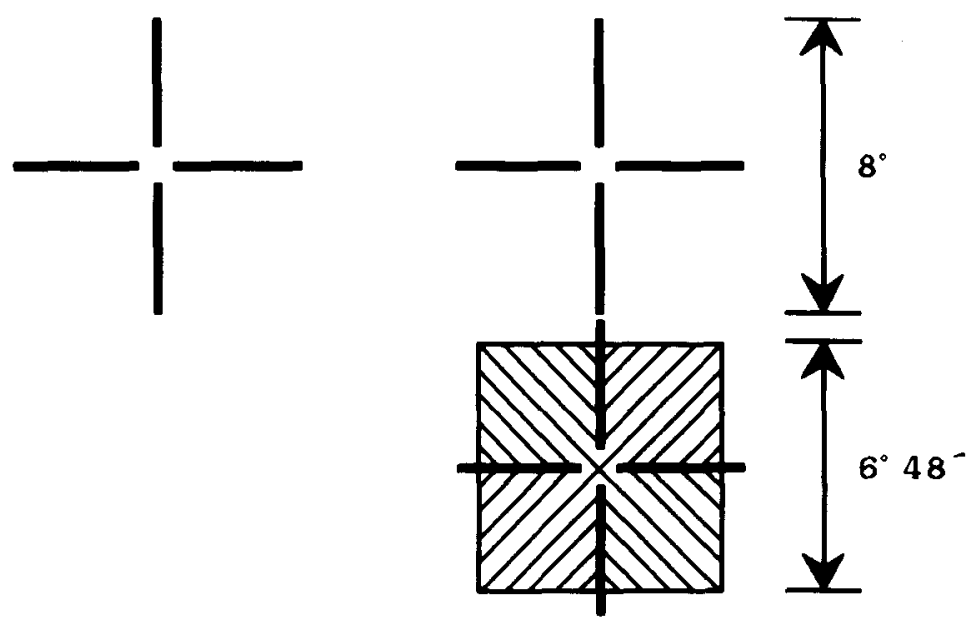

stimulus l (a)
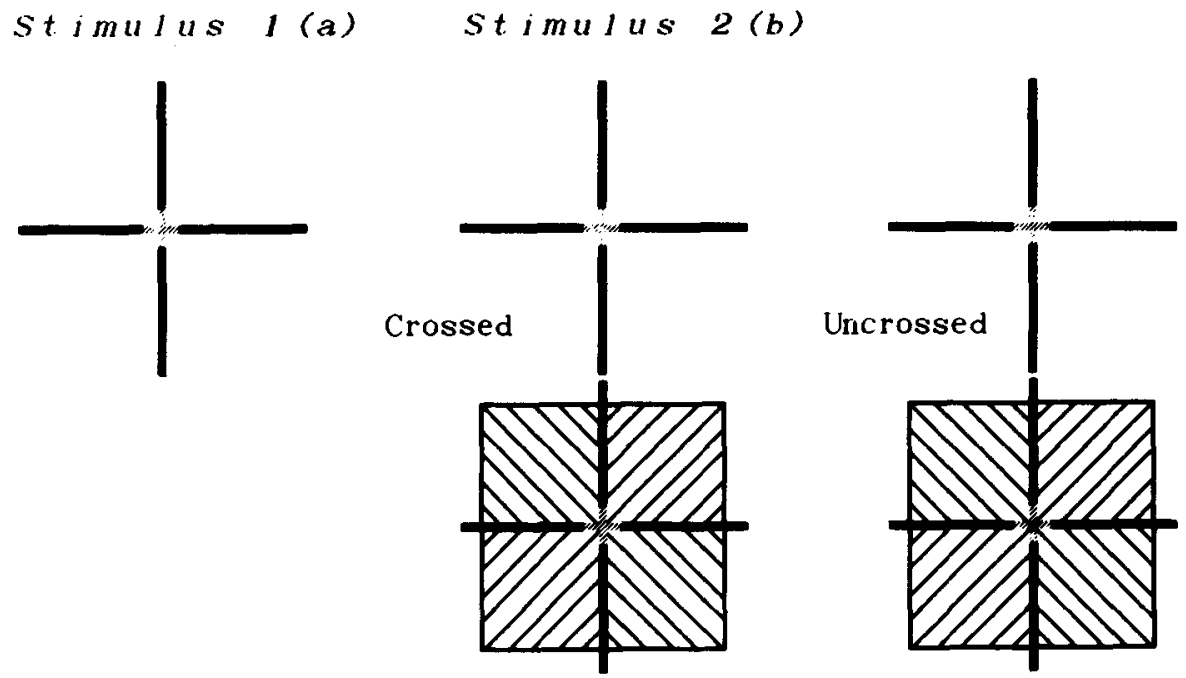

stimulus 3 (c) $s t j m u / u s \quad 4(d 1)$ stimulus 4 (d2)

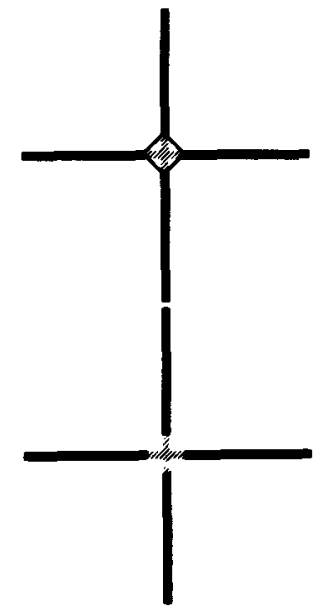

stimulus 5 (e)

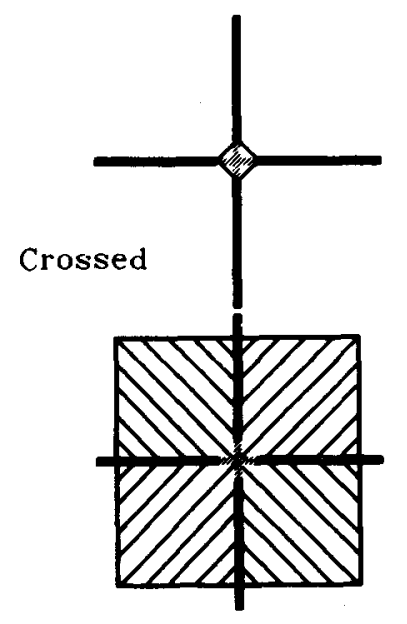

Stimulus $6(t)$

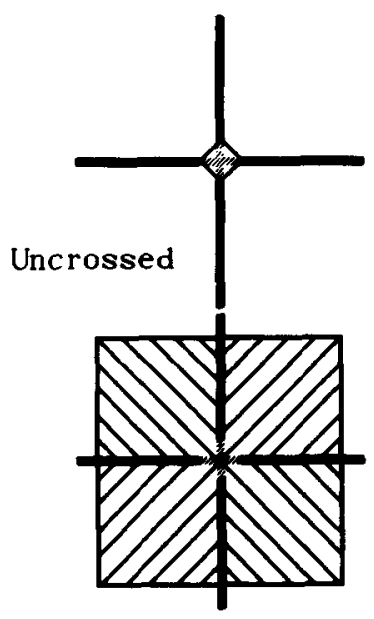

Stimulus $6(t 2)$

Figure 5. Stimuli used in Experiment 2. Stimulus 1-6 (a-f). The four arms of the bottom figures in Stimulus 2 , Stimulus 4 , and Stimulus 6 carry crossed or uncrossed disparity of 18 in real presentations. 
acuity. The authors also served as subjects. The other subjects were not informed of the purpose of the experiment, but 8 of them had served as subjects in the pilot studies of this experiment.

Materials. The stimuli were presented on a CRT display (Commodore 1084) on a microcomputer (Commodore Amiga 500) at a distance of $57.3 \mathrm{~cm}$ from the subjects' eyes.

Six kinds of stimuli were employed, as is shown in Figure 5. All were presented binocularly. Figure 5a shows Stimulus 1, in which an Ehrenstein figure is presented at the top of the screen with no binocular disparity. Figure 5 b shows Stimulus 2 , which is composed of Stimulus 1 plus another Ehrenstein figure with a crossed or uncrossed disparity so as to be seen in front of or behind a grid composed of black lines at the bottom of the screen. Stimulus 3 (Figure 5c) and Stimulus 4 (for a crossed disparity, Figure 5d1; for an uncrossed disparity, Figure $5 \mathrm{~d} 2$ ) are identical to Stimulus 1 and Stimulus 2, respectively, except that the Redies-Spillmann figures are depicted instead of the Ehrenstein figures. Figure 5e shows Stimulus 5, in which one Redies-Spillmann figure with its four junctions connected by a square (a reference figure) is presented at the top of the screen and another Redies-Spillmann figure is presented at the bottom. The two figures have no disparity. Figure $5 f$ shows Stimulus 6, in which the Redies-Spillmann figure at the top of Stimulus 4 was replaced with the reference figure (for a crossed disparity, Figure 5f1; for an uncrossed disparity, Figure 5f2). No fixation points were presented with them.

In order to obtain separate inputs for each eye, as is required for stereopsis, a phase haploscope was used. This altered CRT images destined for the left and right eyes in synchrony with the opening and closing of very fast LCD shutters mounted on spectacles (Haitex Model X-Specs 3D).

The color of the background was light blue $(x=0.21, y=0.18$, luminance $=41.3 \mathrm{~cd} / \mathrm{m}^{2}$ on the screen and $0.75 \mathrm{~cd} / \mathrm{m}^{2}$ through the spectacles). The color of the arms in the Ehrenstein and Redies-Spillmann figures, grids, and the square in the reference figure were all black (luminance $=0.5 \mathrm{~cd} / \mathrm{m}^{2}$ on the screen and $0.03 \mathrm{~cd} / \mathrm{m}^{2}$ through the spectacles). The color of the inserted crosses in the Redies-Spillmann figures was blue $(x=0.17, y=0.10$, luminance $=26.0 \mathrm{~cd} / \mathrm{m}^{2}$ on the screen and $0.51 \mathrm{~cd} / \mathrm{m}^{2}$ through the spectacles). In both the Ehrenstein and the Redies-Spillmann figures, the width and length of the arms subtended $12^{\prime}$ and $3^{\circ} 30^{\prime}$, respectively. The gap size in the Ehrenstein figure and the length of the inserted cross in the Redies-Spillmann figure were both $1^{\circ}$. Thus, the whole length of these two figures was $8^{\circ}$. The whole width and height of the grids were $6^{\circ} 48^{\prime}$. The width of lines constituting the grids was $6^{\prime}$. The centers of figures at the top and bottom in each stimulus were placed $8^{\circ} 18^{\prime}$ apart from each other.

Procedure. The subjects wore spectacles on which LCD shutters were mounted, sat in a chair equipped with a chinrest, and observed the CRT screen in a dark room.
The whole experiment consisted of three conditions, each of which had three trials. On each trial, the subjects were asked to answer the question posed by an experimenter. Soon after each subject's answer, the stimulus for the trial disappeared and was replaced with the new stimulus for the next trial.

In Condition 1, on the first trial, the subjects were presented with Stimulus 1 and asked if they saw a clear contour connecting the end points of the four arms. If the answer was "yes," they were then asked the shape of the area surrounded by the contour. On the second and third trials, Stimulus 2 was presented, and the subjects were asked if they saw as clear a contour connecting the end points of the four arms in the bottom figure as they saw in the top figure.

In Condition 2, on the first trial, the subjects were presented with Stimulus 3 and asked if they saw a clear contour around the color spreading. If the answer was "yes," they were then asked the shape of the area surrounded by the contour. On the second and third trials, Stimulus 4 was presented, and the subjects were asked if they saw as clear a contour around the color spreading in the bottom figure as they saw in the top figure.

In Condition 3, the subjects were presented with Stimulus 5 on the first trial and Stimulus 6 on the second and third trials. On each of the three trials, the subjects were asked whether the area of color spreading was larger than, the same as, or smaller than the $45^{\circ}$ tilted square area drawn in the top reference figure.

In each of the three conditions, there were two combinations: (1) The bottom figure with a crossed disparity was presented on the second trial and that with an uncrossed disparity on the third trial; (2) the bottom figure with an uncrossed disparity was presented on the second trial and that with a crossed disparity on the third trial. There were two combinations with respect to whether Condition 1 or Condition 2 was presented at first. Condition 3 was always presented after them. The numbers of the subjects allocated to each combination are shown in Table 1 . There were no time limits for subjects' responses, although each subject completed the whole experiment within $10 \mathrm{~min}$.

\section{Results}

All the subjects saw illusory contours in the Ehrenstein and Redies-Spillmann figures. In both figures, they reported that the shape of the area surrounded by the contour was a circle or $a 45^{\circ}$ tilted square with four rounded corners.

The numbers of subjects for each response in each condition are shown in Table 2 for each direction of apparent depth, and all significance levels in this section were assessed with Fisher's exact probability test, two-tailed.

Table 1

Numbers of Subjects Allocated to Each Sequence of Stimulus Presentation

\begin{tabular}{|c|c|c|c|c|c|c|c|c|c|}
\hline \multicolumn{9}{|c|}{ Stimulus Presented } & \multirow{2}{*}{$\begin{array}{c}\text { Number } \\
\text { of Subjects }\end{array}$} \\
\hline Trial 1 & Trial 2 & Trial 3 & Trial 4 & Trial 5 & Trial 6 & Trial 7 & Trial 8 & Trial 9 & \\
\hline 1 & $2 \mathrm{C}$ & $2 \mathrm{UC}$ & 3 & $4 \mathrm{C}$ & $4 U C$ & 5 & $6 \mathrm{C}$ & 6UC & 2 \\
\hline 1 & $2 \mathrm{C}$ & $2 U C$ & 3 & 4UC & $4 C$ & 5 & $6 \mathrm{C}$ & 6UC & 1 \\
\hline 1 & 2UC & $2 \mathrm{C}$ & 3 & $4 \mathrm{C}$ & 4UC & 5 & 6UC & $6 \mathrm{C}$ & 2 \\
\hline 1 & 2UC & $2 \mathrm{C}$ & 3 & 4UC & $4 \mathrm{C}$ & 5 & 6UC & $6 C$ & 1 \\
\hline 3 & $4 C$ & $4 U C$ & 1 & $2 \mathrm{C}$ & $2 U C$ & 5 & $6 \mathrm{C}$ & 6UC & 1 \\
\hline 3 & 4UC & $4 C$ & 1 & $2 \mathrm{C}$ & 2UC & 5 & $6 \mathrm{C}$ & 6UC & 2 \\
\hline 3 & $4 \mathrm{C}$ & 4UC & 1 & 2UC & $2 \mathrm{C}$ & 5 & 6UC & $6 C$ & 1 \\
\hline 3 & 4UC & $4 \mathrm{C}$ & 1 & 2UC & $2 \mathrm{C}$ & 5 & 6UC & $6 \mathrm{C}$ & 2 \\
\hline
\end{tabular}

Note-Numbers in the stimulus columns indicate stimulus numbers. The characters $\mathrm{C}$ and $\mathrm{UC}$ that follow the numbers indicate that the bottom figures in the stimulus carried crossed and uncrossed disparities, respectively. 
Table 2

Numbers of Subjects' Responses in Fach Condition

\begin{tabular}{|c|c|c|c|c|c|c|}
\hline \multirow{2}{*}{$\begin{array}{l}\text { Sign of } \\
\text { Disparity }\end{array}$} & \multicolumn{2}{|c|}{$\begin{array}{c}\text { Condition 1 } \\
\text { (Ehrenstein Figure) }\end{array}$} & \multicolumn{2}{|c|}{$\begin{array}{c}\text { Condition 2 } \\
\text { (Redies-Spillmann Figure) }\end{array}$} & \multicolumn{2}{|c|}{$\begin{array}{c}\text { Condition } 3 \\
\text { (Size of Neon Area) }\end{array}$} \\
\hline & Crossed & Uncrossed & Crossed & Uncrossed & Crossed & Uncrossed \\
\hline \multicolumn{7}{|c|}{ Illusory contours } \\
\hline Visible & 1 & 8 & 2 & 8 & & \\
\hline Invisible & 11 & 4 & 10 & 4 & & \\
\hline \multicolumn{7}{|l|}{ Neon area } \\
\hline Global & & & & & 3 & 9 \\
\hline Local & & & & & 9 & 3 \\
\hline
\end{tabular}

Note-In Condition 3, global color spreading denotes a size of neon area larger than the square in the reference figure, and local denotes a size the same as the neon area's or smaller.

In the Ehrenstein figures seen behind the grids in Condition 1 , clear illusory contours were visible to most subjects, while in those seen in front of the grids, the illusory contours were invisible to them $(p<.01)$. Similarly, the Redies-Spillmann figures seen behind the grids in Condition 2, clear illusory contours around the color spreading were visible to most subjects, whereas in those seen in front of the grids, illusory contours were invisible to them $(p<.05)$.

Table 3 shows the numbers of 4 response patterns ( 2 responses in one condition $\times 2$ responses in the other) to the stimuli whose bottom figures have the same direction of depth between the two conditions, for each combination of two conditions (Condition 1 vs. Condition 2, Condition 1 vs. Condition 3, Condition 2 vs. Condition 3). The appearance of the illusory contours in the Ehrenstein figure and in the Redies-Spillmann figure significantly coincided with each other when the apparent depth of the figures relative to the grids varied $(p<.05)$. Coincidence was also found (1) between appearance/disappearance of illusory contours in the Redies-Spillmann figure and the global(larger than the square in the reference figure)/local(same as or smaller than the square in

Table 3

Response Patterns to the Stimuli Whose Bottom Figures Carry the Same Sign of Disparity

\begin{tabular}{|c|c|c|c|}
\hline & \multirow[b]{2}{*}{ Conditions } & \multicolumn{2}{|c|}{ Illusory Contours } \\
\hline & & Visible & Invisible \\
\hline \multicolumn{4}{|c|}{$\begin{array}{c}\text { Ehrenstein Figure with a Grid } \\
\times \text { Redies-Spillmann Figure with a Grid }\end{array}$} \\
\hline Illusory Contours & 1 vs. 2 & & \\
\hline Visible & & 7 & 3 \\
\hline Invisible & & 2 & 12 \\
\hline \multicolumn{4}{|c|}{ Ehrenstein Figure with a Grid } \\
\hline Size of Neon Area & 1 vs. 3 & & \\
\hline Global & & 7 & 5 \\
\hline Local & & 2 & 10 \\
\hline \multicolumn{4}{|c|}{ Redies-Spillmann Figure with a Grid } \\
\hline Size of Neon Area & 2 vs. 3 & & \\
\hline Global & & 8 & 4 \\
\hline Local & & 2 & 10 \\
\hline
\end{tabular}

the reference figure) color spreading $(p<.05)$, and (2) between appearance/disappearance of illusory contours in the Ehrenstein figure and the global/local color spreading $(p<.1)$.

\section{Discussion}

The apparent depth of the figure relative to the interposed grids, which was manipulated as an independent variable in Experiment 2, is an extraneous factor related to depth perception, whereas the misalignment manipulated in Experiment 1 is related to figural structure. These factors are different from each other in quality. Nonetheless, congruent results were obtained in both Experiments 1 and 2 , in that the appearance of the illusory contours in the Ehrenstein figure coincided with that in the Redies-Spillmann figure.

Thus, the hypothesis that the illusory contours seen in the Ehrenstein figure and those seen in the Redies-Spillmann figure are caused by the same mechanism should be more plausible than the rather complicated assumption that the two independent variables that are different in quality by chance have similar effects on the two different illusory contours of different origins.

Several other findings are consistent with the hypothesis suggested by the present study:

First, Redies and Spillmann (1981), manipulating several stimulus parameters, indicated that brightness enhancement in the Ehrenstein figure can be seen whenever the colored inserted cross is removed from the figure in which the neon color spreading is seen, and vice versa.

Second, color spreading with illusory contours was also observed in Varin's (1971) modified Kanizsa (1955) pattern, in which the otherwise incomplete disks are completed by colored sectors. On the one hand, color spreading over the illusory square occurred when the vertical edges of the colored sectors carried a crossed disparity. On the other hand, color spreading did not occur when they carried an uncrossed disparity (Nakayama, Shimojo, \& Ramachandran, in press). This result is analogous to the following observation: On the one hand, the illusory square was seen clearly in Kanizsa's pattern when the vertical edges of the cut sectors carried a crossed disparity; on the other hand, it was no more visible when they car- 

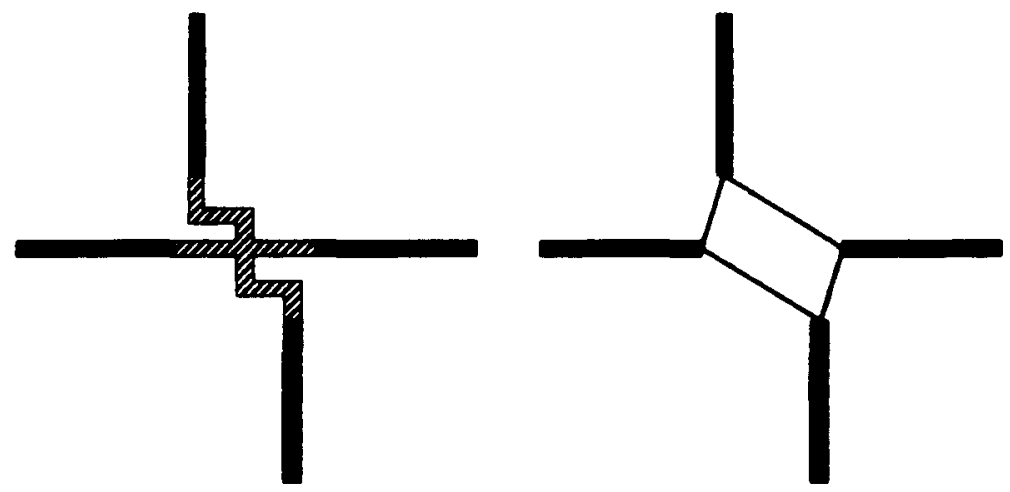

Figure 6. An example of the stimuli used in Condition 1 of Experiment 2. A misaligned Redies-Spillmann figure is shown on the left side, and a misaligned Ehrenstein figure whose four inner tips are connected by a parallelogram is shown on the right. The amount of misalignment was the same in both figures.

ried an uncrossed disparity (Ramachandran, 1986; Ramachandran \& Cavanagh, 1985).

Third, Watanabe and Sato (1989) have found that a luminance difference between the arms and their surroundings is required for the contours surrounding neon color spreading in the Redies-Spillmann figures to be seen. This result is also analogous to the finding that a luminance difference between the inducing figures and their surroundings is required for illusory contours to be seen in figures such as Kanizsa's figure (Cavanagh, 1987; Frisby \& Clatworthy, 1975; Gregory, 1977).

These findings, together with the present results, are consistent with the hypothesis of a common origin of illusory contours seen in the Ehrenstein figure and the Redies-Spillmann figure.

In Experiment 3, in order to confirm the contingency between the appearance/disappearance of illusory contours and global/local color spreading, which was found in Experiment 2, we varied the misalignment and compared the size of color spreading in the Redies-Spillmann figures with the positive response rates of the perception of the illusory contour in the Ehrenstein figures.

\section{EXPERIMENT 3}

\section{Method}

Subjects. Four men, ranging in age between 25 and 31 years, participated as subjects. All of them had served as subjects in Experiment 1 or Experiment 2. They had normal or corrected-tonormal visual acuity. The authors served as subjects, and the other subjects were not aware of the purpose of the experiment.

Materials and Procedure. There were two conditions in Experiment 3. In Condition 1, the procedure and stimuli presented were identical to those in Condition 1 in Experiment 1. In Condition 2, the two figures shown in Figure 6 were presented for each trial. On the left side, a misaligned Redies-Spillmann figure used in Condition 2 in Experiment 1 (Figure 3) was presented at 51' to the left of the center of the display. In a symmetrical position on the right side, the modified Ehrenstein figure whose amount of misalignment was equal to that in the left figure was presented with the four inner tips connected by a parallelogram. ${ }^{3}$ The line of the parallelogram subtended about $2^{\prime}$.
On each trial, the subjects had to report if color spreading seen in the left figure (misaligned Redies-Spillmann figure) was larger than, the same as, or smaller than the area enclosed by the parallelogram in the right figure.

Condition 1 was presented first to 2 of the 4 subjects, and Condition 2 was presented first to the other two. Otherwise, the method was identical to that in Experiment 1.

\section{Results}

Figure 7 shows that the response rates for the perception of the illusory contours decreased, just as in Experiment 1 , while the rates of the response that color spreading was smaller than the parallelogram increased, as the amount of misalignment increased. ${ }^{6}$ The $50 \%$ response rates for both conditions were nearly equal.

The amounts of misalignment corresponding to the 50\% response rate in each condition for each of two orienta-

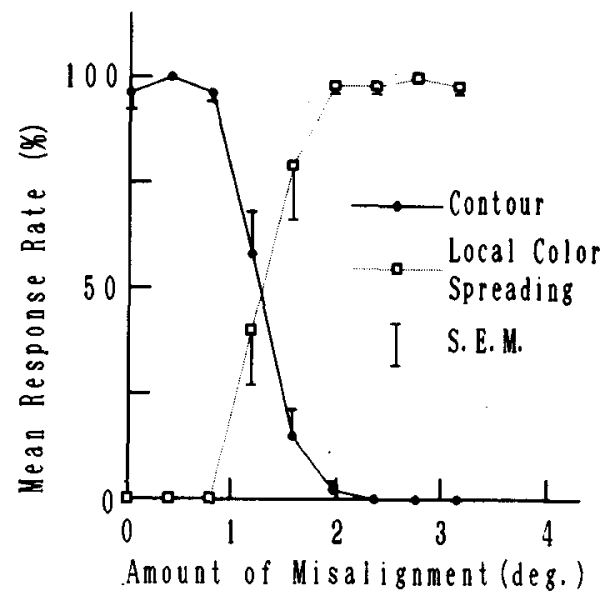

Figure 7. Solid lines represent the mean positive response rates for the perception of illusory contours as a function of the amount of misalignment. Dotted lines represent the mean response rates indicating that color spreading in the Redies-Spillmann figure on the left is smaller than the parallelogram on the right side of the figure. Length of each error bar, which is shown only on one side, represents one standard error of the mean (SEM). 
tions (vertical and horizontal) of misalignment for each subject were subjected to a two-way analysis of variance (ANOVA). The experimental conditons and the orientations of misalignment were the repeated factors. Neither effect was found to be significant.

These results show that color spreading in the modified Redies-Spillmann figure is smaller than the parallelogram when the amount of misalignment is too large for an illusory contour to be seen in the corresponding Ehrenstein figure. Moreover, color spreading in the modified Redies-Spillmann figure is as large as or larger than the parallelogram when the amount of misalignment is small enough for an illusory contour to be perceived in the corresponding Ehrenstein figure.

\section{Discussion}

The results obtained in Experiment 3 are in accordance with those in Experiment 2, in spite of the fact that independent variables are different from each other.

In addition, the coincidence between the appearance/disappearance of the illusory contours and the global/local color spreading in both Experiments 2 and 3 is at odds with the observation of the modified Koffka figure in which the color spreading spreads more extensively without illusory contours than it does with illusory contours.

One possible explanation for the correlation between the range of color spreading and illusory contours found in Experiments 2 and 3 is that there is an interaction between illusory contours and color spreading, by means of which the color spreading is seen to cover the area contained by the illusory contours. In Experiment 4, we added new lines inducing the illusory contours to the line elements inducing local color spreading. We expected that the additional experiment would induce color spreading covering the area surrounded by the illusory contours.

\section{EXPERIMENT 4}

\section{Method}

Subjects. Seven men and 5 women, ranging in age between 19 and 26 years, participated as subjects. All had normal or correctedto-normal visual acuity. None of the subjects were informed of the purpose of the experiment.

Materials. The stimuli were presented on a CRT display (Commodore 1084) of a microcomputer (Commodore Amiga 500) at a distance of $57.3 \mathrm{~cm}$ from each subject's eyes.

Two kinds of stimuli were employed, as is shown in Figure 8a-b. The stimuli consisted of white lines and circles (luminance $=$ $\left.42.5 \mathrm{~cd} / \mathrm{m}^{2}\right)$, blue lines $(x=0.17, y=0.11$, luminance $=$ $27.5 \mathrm{~cd} / \mathrm{m}^{2}$ ), which are represented as gray lines in Figure 8, and a black background (luminance $=0.5 \mathrm{~cd} / \mathrm{m}^{2}$ ). The width of white lines, white circles, and blue lines subtended $6^{\prime}$. The length of shorter and longer white lines subtended $1^{\circ} 20^{\prime}$ and $2^{\circ} 40^{\prime}$. The length of blue lines and diameters of white circles subtended $1^{\circ}$.

Procedure. There were two trials in the whole experiment. The subjects were presented with Figure $8 \mathrm{a}$ or $\mathbf{8 b}$. At first, they were asked whether they saw a clear illusory contour connecting the end points of the arms. Second, they had to report whether or not blue color spread out of the blue lines in the left part of the presented stimulus. If they answered "yes" to the second question, they were further asked whether the range of the color spreading was smaller than, the same as, or larger than the area surrounded by the white circles in the right part of the stimulus. Soon after their responses, the stimulus was replaced with the other one. One half of the subjects were presented Figure $8 \mathrm{a}$ at first and the other half Figure $8 b$ at first. There were no time limits for the subjects' responses. The experiment was conducted in a dark room.

\section{Results and Discussion}

All the subjects saw the clear illusory contour in Figure $8 \mathrm{a}$, whereas no one did in Figure $8 \mathrm{~b}\left[\chi^{2}(1)=12\right.$, $p<.01$, respectively]. All the subjects reported that the blue color spread out of the blue lines in the stimuli represented in Figure 8a-b $\left[\chi^{2}(1)=12, p<.01\right.$, respectively]. For Figure 8a, all the subjects reported that the range of the color spreading was smaller than the circle area $\left[\chi^{2}(2)=24, p<.01\right]$. For Figure $8 b, 11$ subjects reported that the range of color spreading was the same as the circle area $\left[\chi^{2}(2)=18.5, p<.01\right]$, and the remaining subject reported that it was smaller than the circle area.

These results indicate that the color spreading, which was confined to a small area alongside of the colored line adjoined by white lines, was seen to cover the area surrounded by illusory contours when the elements inducing the illusory contour were added. This is compatible with the present hypothesis, according to which there is some interaction between illusory contours and color spreading by means of which the color spreading is seen throughout the area surrounded by illusory contours. There could be no factors contributing to color spreading's being seen throughout the area, other than the addition of line elements with which the necessary condition for the perception of the illusory contours is satisfied.

\section{GENERAL DISCUSSION}

From the results of Experiments 1 and 2, it would be plausible to infer that illusory contours in the Ehrenstein figures and in the Redies-Spillmann figures are caused by the same mechanism. The correlation between the illusory contours and global color spreading shown in the results of Experiments 2 and 3 led to the hypothesis that there is some interaction between illusory contours and color spreading by means of which the color spreading is seen to extend to the illusory contour. This hypothesis is supported by the results of Experiment 4 . Small color spreading was seen to cover the area surrounded by the illusory contours when elements that induce the illusory contours were added.

It is suggested that the color spreading in the Redies-Spillmann figure is originally local, but that as a result of the interaction between the mechanism for illusory contours and that for color spreading, it is seen throughout the area surrounded by the illusory contours.

On the other hand, if color spreading is strong, as in the modified Koffka figure (Day, 1983), illusory contours 

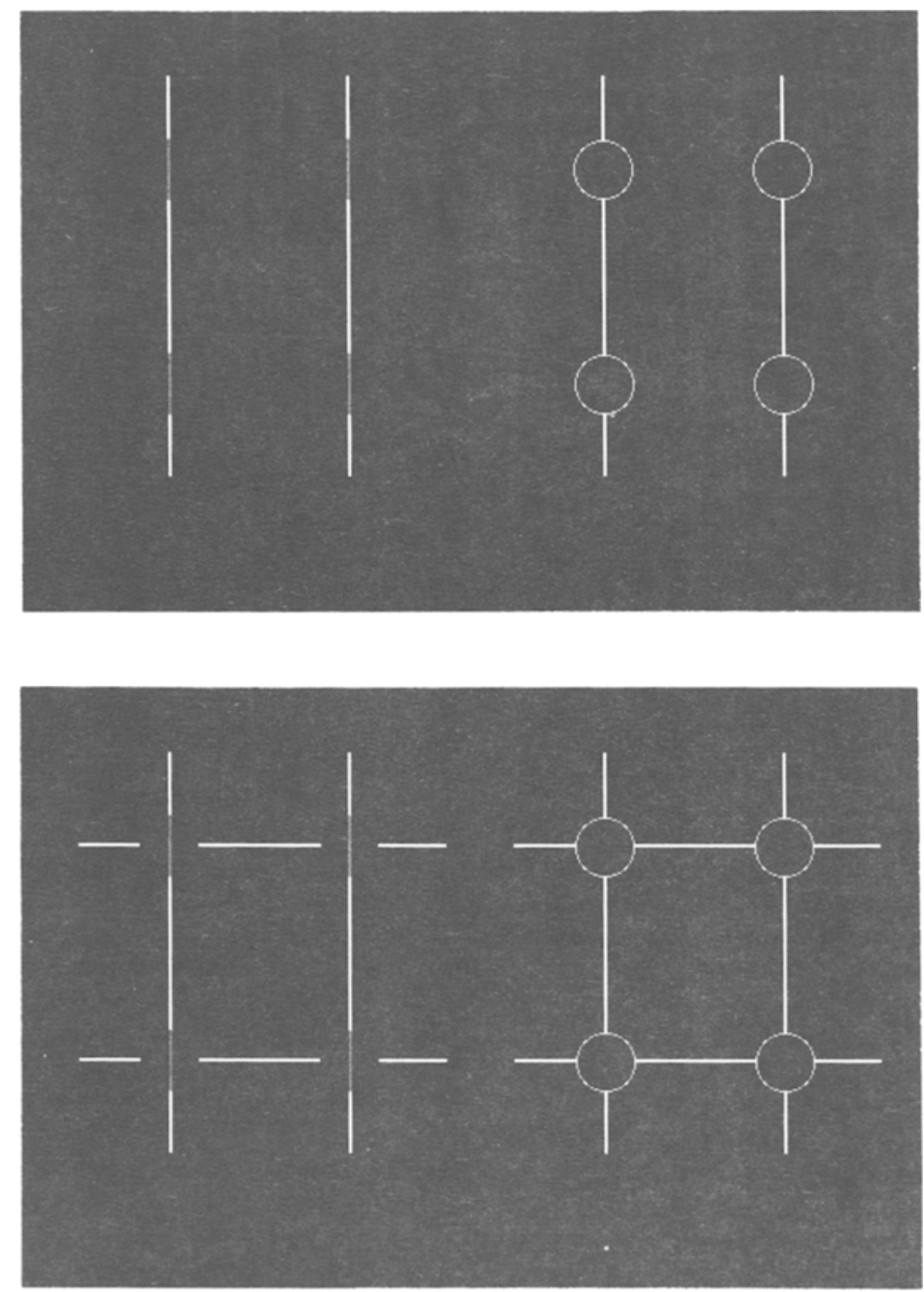

Figure 8. The two stimuli ( $a$ and b) used in Experiment 4. Gray lines represent the colored lines used in the experiment.

may arrest the color spreading. Thus, it is plausible that illusory contours not only arrest color spreading but also cause some interaction with the color spreading.

Grossberg and Mingolla (1985a, 1985b) constructed a model in which color spreading was extended by mutual competition between illusory contours and the contour defining colored cross in the Redies-Spillmann figure. In their model, the contour of the colored cross is weakened by the competition, and its color spreads out of the contour until it reaches the illusory contour. The interaction between color spreading and illusory contours suggested in the present study may be in accordance with their model.

It is noticeable that global brightness enhancement is usually seen throughout the area surrounded by illusory contours in figures such as the Ehrenstein figure and the Kanizsa triangle.
Recently, de Weert and van Kruysbergen (1987) have indicated that global brightness enhancement does not necessarily lead to the perception of sharp illusory contours. Kellman and Loukides (1987) and Prazdny (1983), using black and white inducing elements, found that illusory contours persist in the absence of the global brightness enhancement. On the basis of these findings, they suggest that illusory contours are not a direct result of the global brightness enhancement. Furthermore, using the causal inference method (Blalock, 1962), Watanabe and Oyama (1988) suggested that illusory contour clarity determines the amount of global brightness enhancement, rather than that the latter determines the former.

Several researchers have suggested that a local brightness enhancement occurs only in the vicinity of the inducing elements of the illusory contours at an early stage 
of the formation of the illusory contours (Day \& Jory, 1978, 1980; Frisby \& Clatworthy, 1975; Jory \& Day, 1979; Redies, Spillmann, \& Kunz, 1984). If the interaction suggested in the present study also occurred between illusory contours and the local brightness enhancement, the local brightness enhancement would be seen throughout the area surrounded by the illusory contours and become global brightness enhancement. This assumption is in accordance with the above suggestions that illusory contours are not a direct result of brightness enhancement but that they determine it.

Recently, direct recording of cells has suggested that illusory contours are generated in an early level in visual information processing. Von der Heydt, Peterhans, and Baumgartner (1984) found that primate cells in area 18 responded to a Kanizsa-type illusory bar, whereas cells in area 17 did not. Redies, Crook, and Creutzfeldt (1986) found that $C$ cells in areas 17 and 18 of the cat responded to phase-shift illusory contours. Thus, the suggested interaction between illusory contours and local color spreading might occur at or later than area 17 or 18.

\section{REFERENCES}

BLALOCK, H. M. (1962). Four-variable causal models and partial correlations. American Journal of Sociology, 68, 182-194.

CaVANAGH, P. (1987). Reconstructing the third dimension: Interactions between color, texture, motion, binocular disparity, and shape. Computer Vision, Graphics, \& Image Processing, 37, 171-195.

DAY, R. H. (1983). Neon color spreading, partially delineated borders, and the formation of illusory contours. Perception \& Psychophysics, 34, 488-490.

DAY, R. H., \& JORY, M. K. (1978). Subjective contours, visual acuity and line contrast. In J. C. Armington, J. E. Krauskopf, \& B. Wooten (Eds.), Visual psychophysics: Its physiological basis (pp. 331-340). New York: Academic Press.

DAY, R. H., \& JoRY, M. K. (1980). A note on a second stage in the formation of illusory contours. Perceprion \& Psychophysics, 27, 89-91.

DE WeERT, C. M. M., \& VAN KRUYSBergen, N. A. W. H. (1987). Subjective contour strength and perceptual superimposition: Transparency as a special case. In S. Petry \& G. E. Meyer (Eds.), The perception of illusory contours (pp. 165-170). New York: SpringerVerlag.

Ehrenstein, W. (1941). Über Abwandlungen der L. Hermannschen Helligkeitserscheinung. Zeitschrift fur Psychologie, 150, 83-91. [Modifications of the brightness phenomenon of L. Hermann (1987) (Anne Hogg, Trans.). In S. Petry \& G. E. Meyer (Eds.), The perception of illusory contours (pp. 35-39). New York: Springer-Verlag.]

Ejima, Y., Redies, C., Takahashi, S., \& Akita, M. (1984). The neon color effect in the Ehrenstein pattern: Dependence on wavelength and illuminance. Vision Research, 24, 1719-1726.

Frisby, J. P., \& Clatworthy, J. L. (1975). Illusory contours: Curious cases of simultaneous brightness contrast? Perception, 4, 349-357.

GREGORY, R. L. (1977). Vision with isoluminant colour contrast: 1 . A projection technique and observations. Perception, 6, 113-119.

Grossberg, S., \& Mingolla, E. (1985a). Neural dynamics of form perception: Boundary completion, illusory figures and neon color spreading. Psychological Review, 92, 173-211.

Grossberg, S., \& Mingolla, E. (1985b). Neural dynamics of perceptual grouping: Textures, boundaries, and emergent segmentations. Perception \& Psychophysics, 38, 141-171.

JoRY, M. K., \& DAY, R. H. (1979). The relationship between brightness contrast and illusory contours. Perception, 8, 3-9.

KanIZSA, G. (1955). Margini quasi-percettivi in campi con stimolazione omogenea. Rivista di Psicologia, 49, 7-30. [Quasi-perceptual edges in homogeneously stimulated fields (1987) (Walter Gerbino, Trans.). In S. Petry \& G. E. Meyer (Eds.), The perception of illusory contours (pp. 40-49). New York: Springer-Verlag.]

Kellman, P. J., \& Loukides, M. G. (1987). An object perception approach to static and kinetic subjective contours. In S. Petry \& G. E. Meyer (Eds.), The perception of illusory contours (pp. 151-164). New York: Springer-Verlag.

KoffKA, K. (1935). Principles of Gestalt psychology. New York: Harcourt Brace.

Nakayama, K., Shimojo, S., \& Ramachandran, V. S. (in press). Transparency: Its relation to depth, subjective contours and neon color spreading. Perception.

PrazdNy, K. (1983). Illusory contours are not caused by simultaneous brightness contrast. Perception \& Psychophysics, 34, 403-404.

RAMACHANDRAN, V. S. (1986). Capture of stereopsis and apparent motion by illusory contours. Perception \& Psychophysics, 39, 361-373.

Ramachandran, V. S., \& Cavanagh, P. (1985). Subjective contours capture stereopsis. Nature, 317, 527-530.

Redies, C., Crook, J. M., \& Creutzfeldt, O. D. (1986). Neuronal responses to borders with and without luminance gradients in cat visual cortex and dorsal lateral geniculate nucleus. Experimental Brain Research, 61, 469-481.

Redies, C., \& SpILlmanN, L. (1981). The neon color effect in the Ehrenstein illusion. Perception, 10, 667-681.

Redies, C., Spillmann, L., \& Kunz, K. (1984). Colored neon flanks and line gap enhancement. Vision Research, 24, 1301-1309.

Rock, I., ANSON, R. (1979). Illusory contours as the solution to a problem. Perception, 8, 665-681.

van TUiJl, H. F. J. M. (1975). A new visual illusion: Neonlike color spreading and complementary color induction between subjective contours. Acta Psychologica, 39, 441-445.

VARI, D. (1971). Fenomeni di contrasto e diffusione chromatica nell' organizzazione spaziale del campo percettivo. Rivista di Psicologia, $65,101-128$.

von der Heydt, R., Peterhans, E., \& Baumgartner, G. (1984). Illusory contours and cortical neuron responses. Science, 224, $1260-1262$

Watanabe, T., OYama, T. (1988). Are illusory contours a cause or a consequence of apparent differences in brightness and depth in the Kanizsa square? Perception, 17, 513-521.

Watanabe, T., \& SATo, T. (1989). Effects of luminance contrast on color spreading and illusory contour in the neon color spreading effect. Perception \& Psychophysics, 45, 427-430.

\section{NOTES}

1, The local color spreading without illusory contours was first named "neon flanks" by Redies, Spillmann, and Kunz (1984). In the present study, we do not use the term "neon flanks," since we discuss the extent of color spreading (local/global) and the perception of illusory contours surrounding the color spreading (with/without illusory contours) separately. When color spreads outside of its containing contours and is bounded by a clear illusory contour, we call the phenomenon color spreading with illusory contours. When color spreads with no clear illusory contour, we call the phenomenon color spreading without illusory contours. We use color spreading as a general term to denote the color spread outside of its containing contours irrespective of appearance of illusory contours.

2. Our pilot study indicated that the effect is most salient when the cross is blue (see also Ejima et al., 1984).

3 . Since there was no significant difference between the results for vertical and those for horizontal misalignments, as will be indicated later, the positive response rates on the figures with the same amounts of misalignment, vertically or horizontally, were pooled and are shown in Figure 4. The size of the gap between arms in the Redies-Spillmann figure used in the experiment was $1^{\circ} 36^{\prime}$; and all subjects saw the neon color spreading in the Redies-Spillmann figure without misalignments. However, this gap size exceeds the size for which the neon color spreading is seen both under foveal vision ( $35^{\prime}$ maximum) and extrafoveal vision (about $1^{\circ}$ ) (Redies \& Spillmann, 1981). One possible explanation 
for the apparently incongruous results is that eye movements were permitted in our experiment, and these eye movements could cause the neon color spreading to be seen more easily. In fact, Redies and Spillmann (1981) reported that with eye movements, neon color spreading recurred after the disappearance due to fixation.

4. According to Rock and Anson (1979), the grids seen behind the inducing figures lead to the impression that the central region of the illusory contour display is transparent; this illusory surface covering the comer fragments should be opaque, however. Rock and Anson suggested that this contradiction removes the illusory surface. The illusory surface in the Redies-Spillmann figure does not look opaque but rather transparent (Nakayama, Shimojo, \& Ramachandran, in press; Redies \& Spillmann, 1981). Therefore, if Rock and Anson's suggestion is true, the neon color spreading would persist even when the Redies-Spillmann figure is seen in front of the grids.
5. Subjects reported that the illusory contours, if seen, occurred along the parallelogram connecting the four line ends or along the tilted ellipse that circumscribes the parallelogram in the modified Ehrenstein figure. That is, the parallelogram was the smallest area in which illusory contours were seen with each amount of misalignment.

6. Since there was no significant difference between the results for vertical and those for horizontal misalignments, the positive response rates on the figures with the same amount of misalignment, vertically or horizontally, were pooled and are shown in Figure 7, as was also done in Figure 4.

(Manuscript received April 3, 1989; revision accepted for publication December 12, 1989.) 